\title{
Thermal fracture characteristics induced by laser beam
}

\author{
Y.C. Zhou ${ }^{\mathrm{a}, \mathrm{b}, \mathrm{c}, *}$, Z.M. Zhu ${ }^{\mathrm{a}}$, Z.P. Duan ${ }^{\mathrm{b}}$ \\ a Institute of Fundamental Mechanics and Material Engineering, Xiangtan University, Xiangtan, Hunan 411105, People's Republic of \\ China \\ ${ }^{\mathrm{b}}$ Institute of Mechanics, Chinese Academy of Sciences, Beijing, 100080, People's Republic of China \\ ${ }^{\mathrm{c}}$ Fracture Research Institute, Tokoku University, 01 Aza-Aoba, Aramaki, Aobaku, Sendai 980-8579, Japan \\ Received 21 April 2000
}

\begin{abstract}
The damage mechanism of a cracked material due to laser beam thermal shock is an important problem when the interactions of high power laser beam with materials are studied. The transient thermal stress intensity factors (TSIFs) for a center crack in an infinite plate subjected to laser beam thermal shock are investigated. When the crack is in the heat affected zone, the compressive thermal stress causes the crack surface to come into contact with each other over a certain contact length, but the high tensile stresses around the crack tip tend to open the crack. In this case, the problem may be treated as a pair of embedded cracks problem with a smooth closure condition of the center crack. The TSIFs and the crack contact lengths are calculated with different laser beam spatial shapes. The TSIFs induced by thermal shock are in marked different from those induced by general mechanical loading. ( $) 2001$ Elsevier Science Ltd. All rights reserved.
\end{abstract}

Keywords: Thermal fracture; Thermal shock; Laser beam

\section{Introduction}

In aerospace engineering many structural components may be subjected to severe thermal loading which may be produced by aerodynamic heating, by laser irradiation, or by localized intense fire (e.g., Berlin et al., 1992; Blisset et al., 1997; Kagawa, 1997; Zhou et al., 1998, 1999). However, in the previous studies on laserinduced material damage, one ignored more or less the fact that potential flaws may exist in components. The effect of pre-existing flaws and rapid heating environment must be considered in accurate damage tolerance analysis. If there are defects in such structures, the bodies subjected to laser beam thermal shock will successively undergo a variety of rheological processes. Such processes may be the absorption of light energy, temperature rise, thermal viscoplastic deformation, energy dissipation, intense thermal stress concentration around defects, the growth and coalescence of defects as well as the degradation of strength

\footnotetext{
${ }^{*}$ Corresponding author. Address: Fracture Research Institute, Tokoku University, 01 Aza-Aoba, Aramaki, Aobaku, Sendai 9808579, Japan. Tel.: +81-22-217-7519; fax: +81-22-217-4311.

E-mail address: zhou@rift.mech.tohoku.ac.jp (Y.C. Zhou).
} 
properties at elevated temperature. The non-linear coupled effects of these rheological processes may result in catastrophic failure. The damage analysis for bodies with defects exposed to intense thermal shock is very important for determining the safety of materials, components, especially the aerospace structural components.

Many papers have been written on thermal shock problems. Gupta (1972) determined the strength degradation and crack propagation in thermally shocked $\mathrm{Al}_{2} \mathrm{O}_{3}$. Schneider and Petzow (1991) developed a new testing method to determine the thermal shock fracture toughness $K_{\mathrm{c}}^{\mathrm{TS}}$ of $\mathrm{Si}_{3} \mathrm{~N}_{4}$ up to $1000^{\circ} \mathrm{C}$. Zhou et al. (1998) studied the thermal failure of SiC particulate reinforced 6061 aluminum alloy composites induced by the coupled loading both with laser thermal shock and general mechanical load. The characteristics of thermal fracture induced by laser beam are different from those induced by general mechanical load.

To understand the characteristics of thermal fracture induced by laser beam, transient thermal stress intensity factors (TSIFs) for an infinite plate with a center crack subjected to a laser beam thermal shock are investigated as illustrated in Fig. 1. In the analysis, it is assumed that the problem is quasi-static, the thermo-elastic coupled effects and the temperature dependence of thermo-elastic constants are neglected. The temperature and thermal stress fields are first analyzed for the infinite plate with non-crack. The characteristics of thermal stress, which are compressive, will make the center crack be contact and consequently, the center crack will become a pair of embedded cracks as illustrated in Fig. 1(c). The weight function method is used to calculate the TSIFs for the embedded cracks.

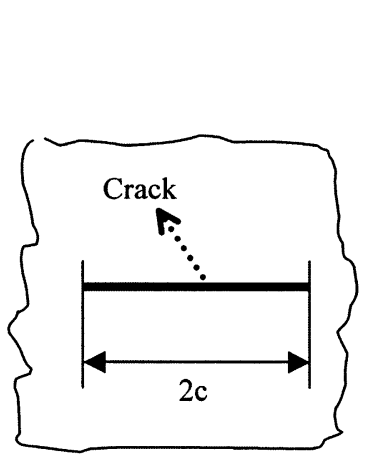

(a)

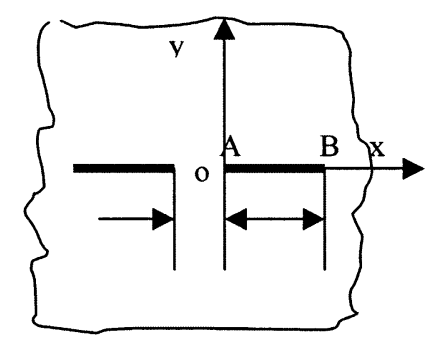

(d)

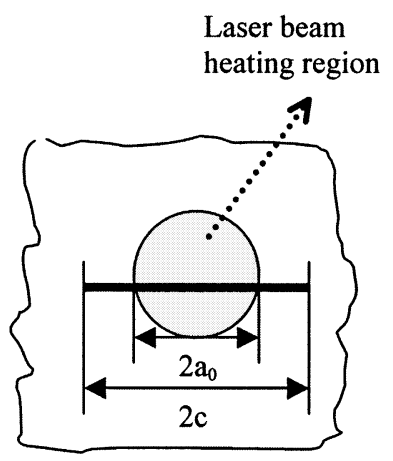

(b)

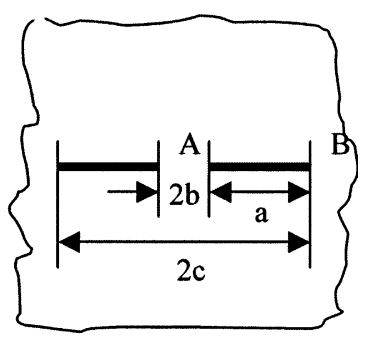

(c)

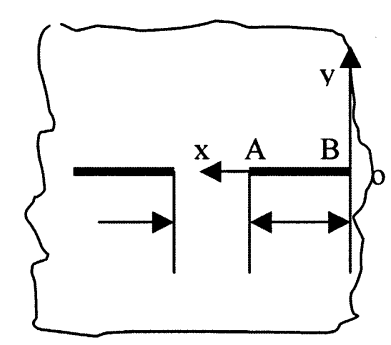

(e)

Fig. 1. A center crack of $2 c$ length in an infinite plate subjected to laser beam thermal shock: (a) center crack geometry; (b) center crack heated by laser beam; (c) the heated center crack with crack contact length $2 b$; (d) and (e) the coordinate system for the coordinate transformation. 


\section{Description of the problem}

\subsection{Temperature field and thermal stress calculation}

The formulation of the crack problem (Fig. 1(a)) makes use of the transient thermal stress from the uncracked problem due to laser beam heating. The transient temperature distribution can be determined by solving the diffusion equation,

$$
\rho c_{\mathrm{p}} \frac{\partial \theta}{\partial t}=\kappa\left(\frac{\partial^{2} \theta}{\partial r^{2}}+\frac{1}{r} \frac{\partial \theta}{\partial r}\right)+\frac{I(r, t)}{h}
$$

under the initial and boundary conditions:

$$
\theta(r, 0)=0 \quad \frac{\partial \theta(0, t)}{\partial r}=0 \quad \theta(\infty, t)=0
$$

where $r$ is the coordinate in radial direction, $t$ is time, $\theta(r, t)=T(r, t)-T_{0}$ is the temperature rise, $T(r, t)$ and $T_{0}$ are the absolute temperature and room temperature, respectively. In above formula, $\kappa, \rho, c_{\mathrm{p}}$ and $h$ are the thermal conductivity, density, specific heat capacity of materials and the thickness of the plate, respectively. The laser beam power intensity $I(r, t)$ absorbed by the material, including its temporal and spatial shapes, is approximated by (Duan et al., 1994; Zhou and Duan, 1996),

$$
I(r, t)=I_{\max } \mathrm{e}^{-\alpha_{1} t}\left(1-\mathrm{e}^{-\beta_{1} t}\right) f(r)=I_{\max } g(t) f(r)
$$

where $\alpha_{1}$ and $\beta_{1}$ are determined experimentally and are equal to $1.5 \times 10^{4}$ and $8.0 \times 10^{4} \mathrm{~s}^{-1}$, respectively. $I_{\max }$ is the maximum value of laser power intensity. In expression (3), $f(r)$ represents the spatial shape of the laser beam and can be expressed by the following three forms,

Shape 1: uniform distribution

$$
f(r)= \begin{cases}1, & \text { if } 0 \leqslant r \leqslant a_{0} \\ 0, & \text { if } a_{0}<r<\infty\end{cases}
$$

Shape 2: Gaussian distribution

$$
f(r)=\mathrm{e}^{-\left(r / a_{0}\right)^{2}} \quad 0 \leqslant r<\infty
$$

Shape 3: normal distribution

$$
f(r)=\left\{\mathrm{e}^{-\left(r_{0} / a_{0}\right)^{2}}+\frac{r_{0}}{a_{0}} \sqrt{\pi}\left[1+\operatorname{erf}\left(\frac{r_{0}}{a_{0}}\right)\right]\right\}^{-1} \mathrm{e}^{-\left(r-r_{0} / a_{0}\right)^{2}} \quad 0 \leqslant r<\infty
$$

where $a_{0}$ is the laser spot radius, $r_{0}$ parameter and $\operatorname{erf}(x)$ error function.

Thermal shock is generated by irradiating a laser beam on the center of the plate. This causes much higher temperatures in the center region of the plate than those in outer region. The higher thermal expansion in the plate center is constrained by the cooler edge. It will induce a tensile hoop stress at the edge, tangential and radial compressive stress in the center. The linear elastic solution for radial and tangential thermal stresses $\sigma_{r r}(r, t)$ and $\sigma_{\theta \theta}(r, t)$ has the following form (Boley and Weiner, 1985),

$$
\sigma_{r r}(r, t)=-\frac{\alpha E}{r^{2}} \int_{0}^{r} \theta(\xi, t) \xi \mathrm{d} \xi, \quad \sigma_{\theta \theta}(r, t)=\alpha E\left[\frac{1}{r^{2}} \int_{0}^{r} \theta(\xi, t) \xi \mathrm{d} \xi-\theta(r, t)\right]
$$

where the temperature-independent Young's modulus and thermal expansion coefficient are denoted by $E$ and $\alpha$, respectively. 
For convenience of numerical calculations, the following dimensionless quantities are introduced,

$$
\rho=\frac{r}{c}, \quad t^{*}=\frac{D t}{c^{2}}, \quad \theta^{*}=\frac{\theta}{\theta_{m}^{0}}, \quad \sigma_{\theta \theta}^{*}=\frac{\sigma_{\theta \theta}(r, t)}{\alpha E \theta_{m}^{0}}, \quad a_{1}=\frac{I_{\max }}{I_{\max }^{0}}, \quad a_{2}=\frac{a_{0}}{c}, \quad r_{0}^{*}=\frac{r_{0}}{c}
$$

where $2 c$ is the length of the center crack, $D=\kappa / \rho c_{\mathrm{p}}$, the reference temperature is $\theta_{m}^{0}=I_{\max }^{0} c^{2} / h \kappa$ and the reference laser power intensity is $I_{\max }^{0}$.

\subsection{Description of the problem}

Transient temperature and thermal stress distributions in the uncracked plate are shown in Figs. 2 and 3, respectively. Fig. 2 shows the transient temperature distributions for different laser spatial shapes at different time. The spatial profile of temperature field is influenced definitely by the spatial shape of laser beam. Different types of laser beam lead to different temperature distributions even though the net energy and duration are same. The tangential thermal stresses $\sigma_{\theta \theta}(r, t)$ corresponding to the temperature fields shown in Fig. 2 are plotted in Fig. 3. From the results shown in Fig. 3, one can see that tangential thermal

(a)

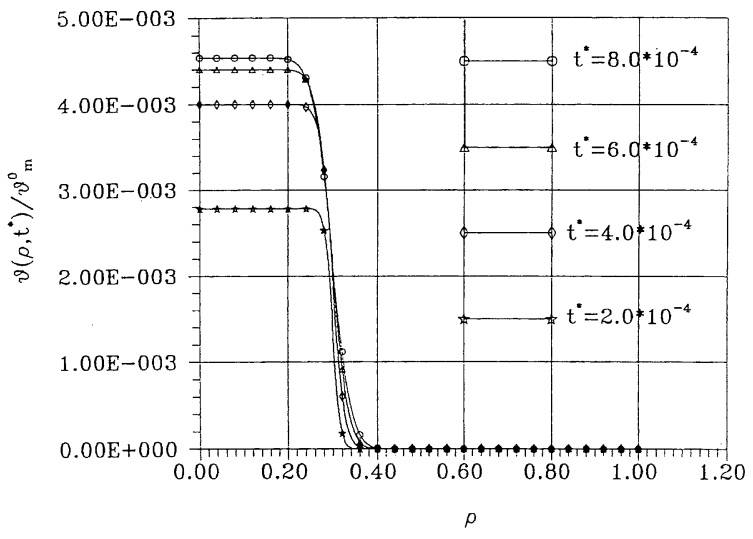

(b)

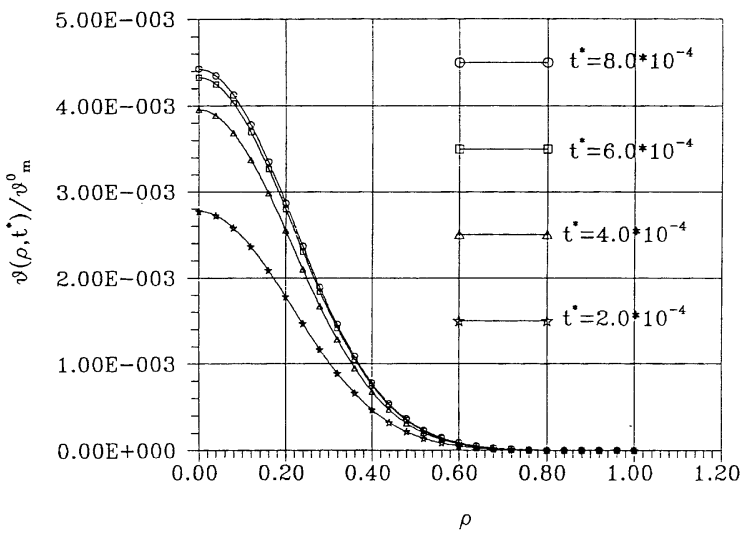

(c)

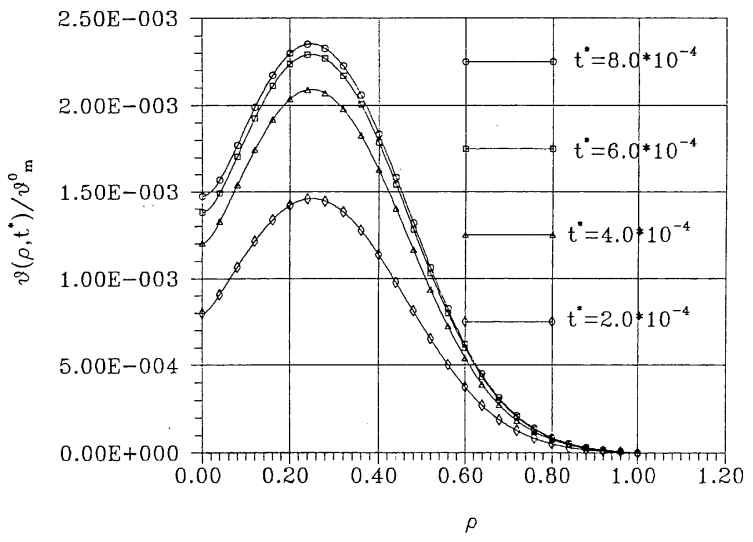

Fig. 2. Transient temperature distributions for different laser spatial shapes at different time: (a) shape 1; (b) shape 2; (c) shape 3, where $a_{1}=30, a_{2}=0.3$ and $r_{0}^{*}=0.25$. 
(a)

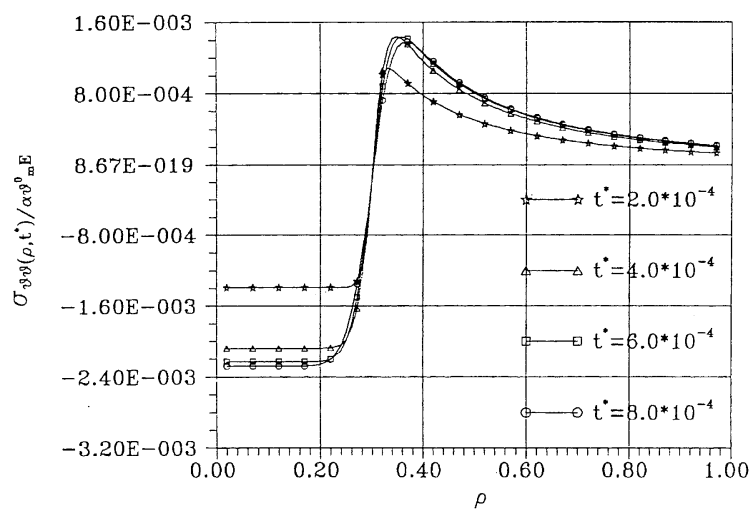

(b)

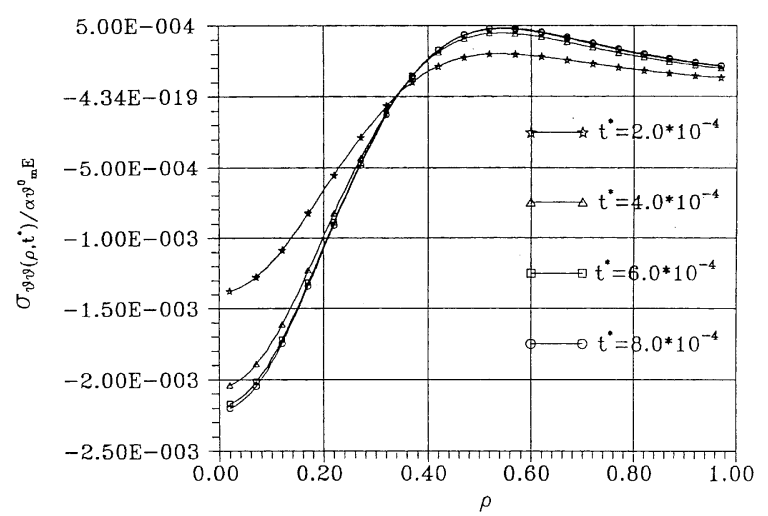

(c)

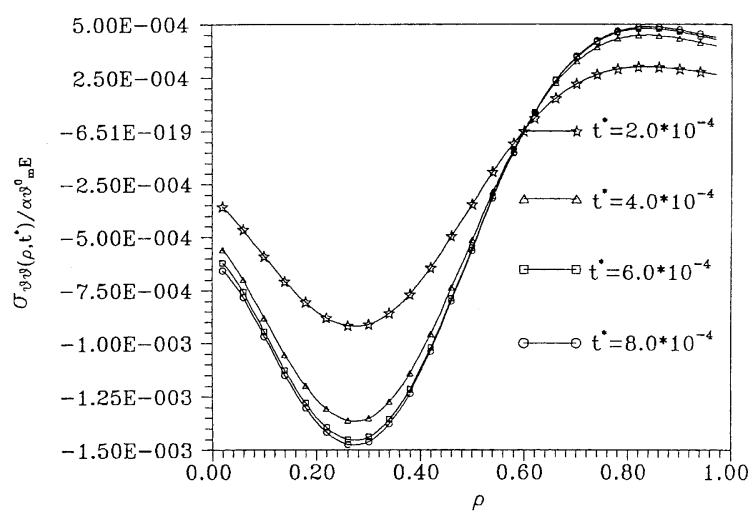

Fig. 3. Corresponding transient tangential thermal stresses $\sigma_{\theta \theta}(r, t)$ to the temperature fields in Fig. 2: (a) shape 1; (b) shape 2; (c) shape 3 , where $a_{1}=30, a_{2}=0.3$ and $r_{0}^{*}=0.25$.

stresses $\sigma_{\theta \theta}(r, t)$ remain negative values within laser spot region and positive values around laser spot region, respectively. Although the circles of zero tangential stresses are different for different laser spatial shapes, the respective circle of zero tangential stresses remains constant. This phenomenon is a special feature of laser beam irradiation.

Only tangential thermal stresses $\sigma_{\theta \theta}(r, t)$ have contribution to crack open or contact. From Fig. 3, one can conclude that the compressive thermal stresses $\sigma_{\theta \theta}(r, t)$ within the heat-affected zone will induce the crack surface to come into contact with each other over a certain contact length $2 b$. The tensile stresses around the edge region of the heat-affected zone may tend to open the crack. In this case, the problem may be treated as a pair of embedded cracks problem with a smooth closure condition of the center crack as illustrated in Fig. 1(c). The crack will propagate if the TSIF is high enough to exceed the critical stress intensity factor at the crack tip.

\subsection{Weight function and thermal stress intensity factors}

The weight function method proposed by Bueckner (1970) and Rice (1972) has proved to be a very useful and versatile method of calculating stress intensity factors, especially for cracks subjected to non-uniform stress fields. Once the weight function $m(x, a)$ for a particular cracked body is determined, the stress 
intensity factor can be calculated by integrating the weight function $m(x, a)$ and the stress distribution $\sigma(x)$ acting in the prospective crack plane. This results in the following express for the stress intensity factor $K$,

$$
K=\int_{0}^{a} \sigma(x) m(x, a) \mathrm{d} x
$$

where $a$ is the crack length. The stress field $\sigma(x)$ has to be determined for uncracked body using an analytical, numerical or experimental method of stress analysis. It was proved by Bueckner (1970) and Rice (1972) that the weight function $m(x, a)$ for a crack in mode I is a unique property of geometry and it can be written in the form,

$$
m(x, a)=\frac{E}{K_{\mathrm{r}}} \frac{\partial u_{\mathrm{r}}}{\partial a}
$$

for plane stress problem of interest. In order to derive the weight function $m(x, a)$ a reference stress intensity factor $K_{\mathrm{r}}$ for a given geometry system under any applied stress needs to be known together with the corresponding crack opening displacement field $u_{\mathrm{r}}(x, a)$. A suitable reference stress intensity factor $K_{\mathrm{r}}$ can often be found in the literature. Petroski and Achenbach (1978) derived a universal crack opening function, which can be used for the calculation of crack opening displacements $u_{\mathrm{r}}(x, a)$ for plane stress problems,

$$
u_{\mathrm{r}}(x, a)=\frac{\sigma_{0}}{\sqrt{2} E}\left[4 F(a) \sqrt{a(a-x)}+\frac{G(a)(a-x)^{3 / 2}}{\sqrt{a}}\right]
$$

where $\sigma_{0}$ is a characteristic stress or nominal stress, $F(a)$ is the parameter of the crack opening displacement function which is given in Appendix A. The function $G(a)$ is also given in Appendix A.

Based on the above theoretical background, one can easily obtain the weight function $m(x, a)$ for the embedded cracks problem (Fig. 1(c)). The reference stress intensity factors in mode I at the crack tips A and B for a pair of embedded cracks problem (Fig. 1(c)) are known as (Tada et al., 1973),

$$
K_{\mathrm{IAr}}=\sigma_{0} \sqrt{\pi b} \frac{c^{2} \frac{E\left(k_{0}\right)}{K\left(k_{0}\right)}-b^{2}}{b \sqrt{c^{2}-b^{2}}} \quad K_{\mathrm{IBr}}=\sigma_{0} \sqrt{\pi c} \frac{1}{k_{0}}\left[1-\frac{E\left(k_{0}\right)}{K\left(k_{0}\right)}\right]
$$

where $2 b$ is the crack contact length. When the crack contact length $2 b$ is introduced into the formulation, the problem is treated as a non-linear system, which must be solved iteratively. In formula (12), $k_{0}, E\left(k_{0}\right)$ and $K\left(k_{0}\right)$ are given in Appendix A. From formulas (11) and (12), after obtaining the parameters of the crack opening displacement function $F_{\mathrm{IAr}}(a)$ and $F_{\mathrm{IBr}}(a)$ at the crack tips $\mathrm{A}$ and $\mathrm{B}$, and the crack opening displacements $u_{\mathrm{rA}}$ and $u_{\mathrm{rB}}$ at the crack tips $\mathrm{A}$ and B, one can obtain the following TSIFs $K_{\mathrm{IA}}$ and $K_{\mathrm{IB}}$ as,

$$
\begin{aligned}
& K_{\mathrm{IA}}=-\frac{1}{\sqrt{2 \pi a} F_{\mathrm{IAr}}(a)} \frac{\partial}{\partial a} \int_{0}^{a}\left[4 F_{\mathrm{IAr}}(a) \sqrt{a(a-x)}+\frac{1}{\sqrt{a}} G_{\mathrm{IAr}}(a)(a-x)^{3 / 2}\right] \sigma_{\theta \theta}^{\mathrm{A}}(x, t) \mathrm{d} x \\
& K_{\mathrm{IB}}=-\frac{1}{\sqrt{2 \pi a} F_{\mathrm{IBr}}(a)} \frac{\partial}{\partial a} \int_{0}^{a}\left[4 F_{\mathrm{IBr}}(a) \sqrt{a(a-x)}+\frac{1}{\sqrt{a}} G_{\mathrm{IBr}}(a)(a-x)^{3 / 2}\right] \sigma_{\theta \theta}^{\mathrm{B}}(x, t) \mathrm{d} x
\end{aligned}
$$

The related functions such as $F_{\mathrm{IAr}}(a), F_{\mathrm{IBr}}(a), u_{\mathrm{rA}}$, and $u_{\mathrm{rB}}$ are given in Appendix A. To obtain $\sigma_{\theta \theta}^{\mathrm{A}}(x, t)$ and $\sigma_{\theta \theta}^{\mathrm{B}}(x, t)$, the abscissa $x$ in the tangential hoop stresses $\sigma_{\theta \theta}(r, t)$ have to be transformed to $x=a+b-r$ and $x=r-b$, respectively.

The formulation of the crack contact problem should depend on the crack contact length $2 b$ in the compressive zone, which is an additional unknown variable. The physical condition, which accounts for this unknown variable is the smooth closure condition of the crack surfaces at crack tip A (Bakioglu et al., 1976; Fig. 1(c)), i.e., $K_{\mathrm{IA}}=0$. Consequently, the calculation of the crack contact zone $2 b$ can be obtained from the solution of the embedded cracks problem by fixing the crack length $2 c$ and then determining 
iteratively the location of the crack tip A (Fig. 1(c)) at each time step such that equation $K_{\mathrm{IA}}=0$ is satisfied. In this manner, the crack contact zone $2 b$ and the stress intensity factor $K_{\mathrm{IB}}$ at crack tip $\mathrm{B}$ for various laser beam thermal shock can be determined.

\section{Numerical results and discussions}

The normalized transient TSIFs at crack tip B and the ratio of the contact length $2 b$ to the overall crack $2 c$ are calculated. The normalized TSIF $K_{\mathrm{B}}^{*}$ is defined by,

$$
K_{\mathrm{B}}^{*}=\frac{K_{\mathrm{B}}}{\alpha E \theta_{m}^{0} \sqrt{c}}
$$

\subsection{The evolution of TSIFs and the dependence of TSIFS on the spatial shape of laser beam}

Fig. 4 contains plots of the normalized transient TSIFs as a function of non-dimensional time $t^{*}$ for different normalized laser intensity $a_{1}$. In Fig. 4, it can be seen that the TSIF increases with the increase of time. After it reaches a maximum value TSIF will decrease. It should also be noted that the maximum TSIF increases with increasing the normalized laser intensity $a_{1}$. Fig. 5 shows the normalized TSIFs versus $t^{*}$ for different normalized laser spot radius $a_{2}$. Fig. 6 shows the normalized TSIFs with $t^{*}$ for different shapes of laser beam, where $a_{1}$ and $a_{2}$ are constant. In Fig. 5, it can be seen that the magnitude of the TSIFs will reduce with the increase of $a_{2}$. From the comparison of the TSIFs for different laser beam shapes in Fig. 6, it can be seen that the TSIFs for shape 1 are much large than those for shapes 2 and 3. This means that the laser beam with spatial shape 1 is more dangerous than that with spatial shapes 2 and 3 . The present investigation confirms that the mode of laser-induced material failure could be altered essentially by changing spatial shape of laser beam when its average parameters such as wavelength, intensity, duration and spot size remained unchanged. In fact, the experimental study on the laser-induced material failure confirms that the failure mode could also be altered by changing spatial shape of the laser beam. The spatial shape of laser beam is generally Gaussian form. But when the spatial shape is an uniform form, a new phenomenon was observed in our experiment and the phenomenon is very different from the general failure mode (Zhou
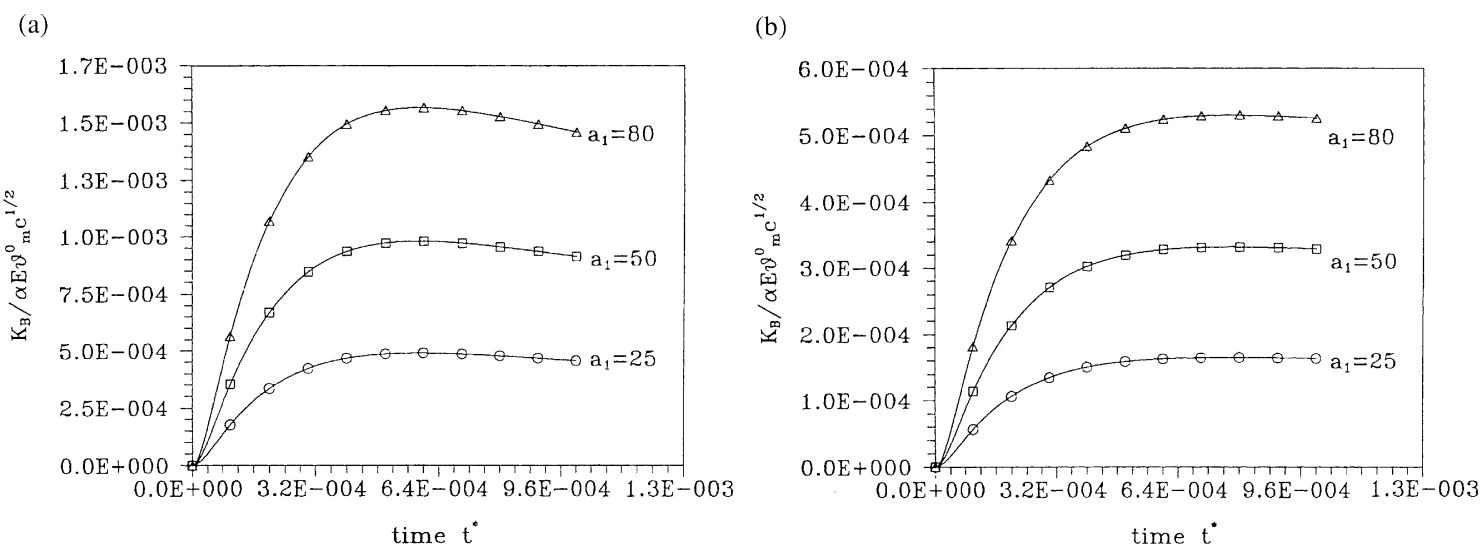

Fig. 4. The curves of normalized transient TSIFs versus normalized time for different normalized laser intensity $a_{1}$ and constant $a_{2}=0.25$ : (a) shape 1 ; (b) shape 2 . 
(a)

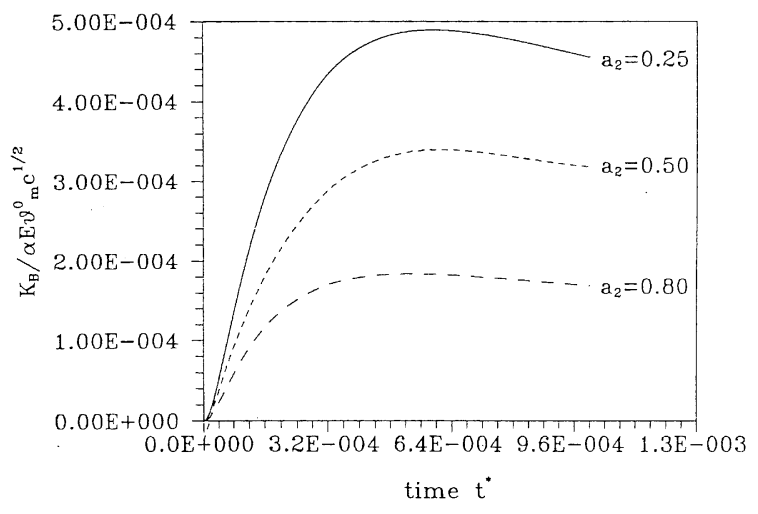

(b)

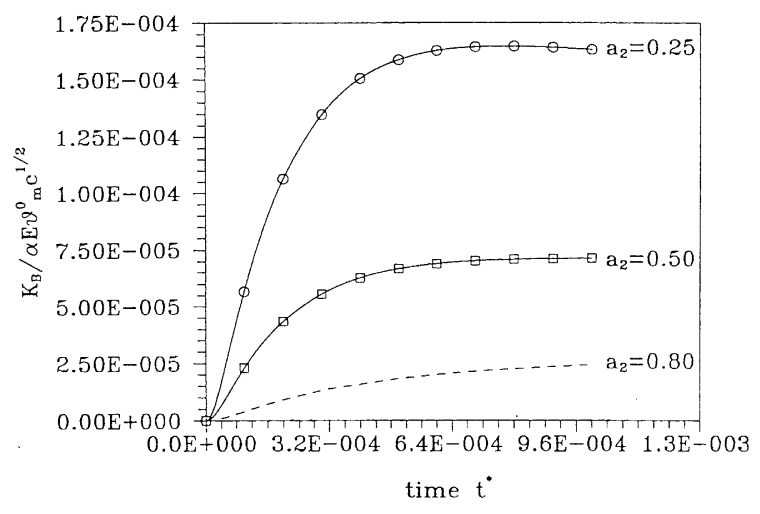

(c)

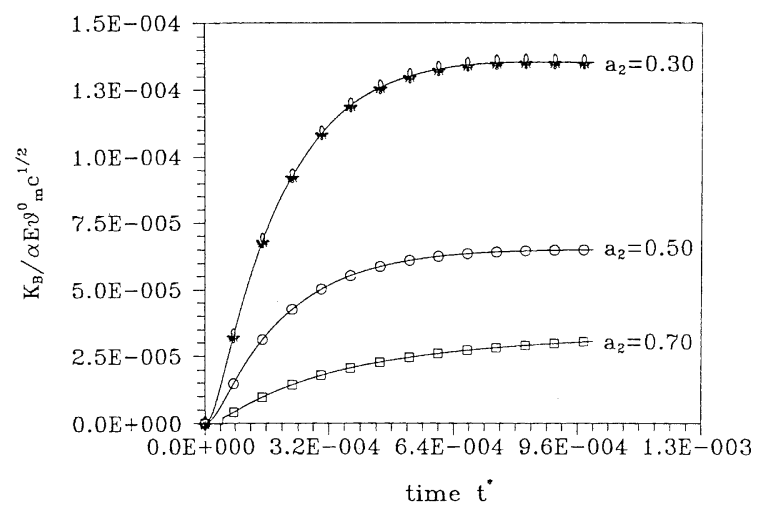

Fig. 5. The variation of the TSIFs $K_{\mathrm{B}}^{*}$ with $t^{*}$ for different normalized laser spot radius $a_{2}$ : (a) shape 1; (b) shape 2 and (c) shape 3 , where $a_{1}=25$ and $r_{0}^{*}=0.1$.

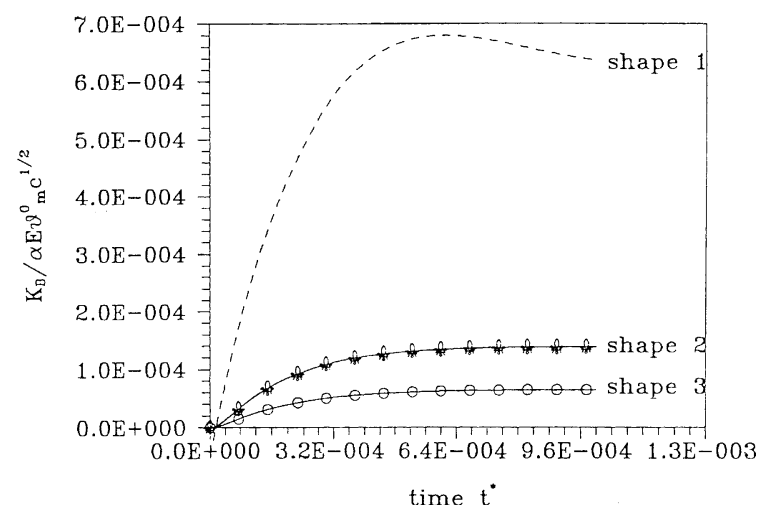

Fig. 6. The curves of normalized transient TSIFs versus normalized time for different laser beam shapes, where $a_{1}=50, a_{2}=0.5$ and $r_{0}^{*}=0.1$. 
and Duan, 1996; Zhou et al., 1999). The distinct phenomenon is reverse bulging and plugging in circular brass foil induced by an uniform spatial shape laser beam.

\subsection{The crack-length-dependent thermal stress intensity factors}

The normalized laser beam spot radius $a_{2}$ has an effect on transient TSIFs and it is shown in Fig. 7. From Fig. 7(a) and (b), one can see that the normalized TSIFs will decrease with the increase of $a_{2}$. The relation of normalized TSIFs with $a_{2}$ is approximately linear as shown in Fig. 7(a) and (b). However, from Fig. 7(c), one can see that the TSIFs decline very sharply with the increase of $a_{2}$ for small laser spot radius, i.e., $a_{2}<0.3$. But for large laser spot radius, i.e., $a_{2}>0.3$, the TSIFs decrease very slowly with the increase of $a_{2}$. For constant laser spot radius, the crack-length-dependent TSIFs are shown in Fig. 8. In Fig. 8(a) and (b), the maximum value of TSIF over the crack length curve separates the plane of TSIF with crack-length to two regions. One is a stable crack-growth region and another is an unstable crack-growth region. This phenomenon is very different from that case in general mechanical load, where external forces are applied and the SIF increases with the square root of the crack length. The crack length $c_{m}$ that corresponds to the maximum TSIF $K_{m}$ decreases with the increase of time. This result is different from that in quenching experiments where $c_{m}$ increase with the increase of time (Oliveira and $\mathrm{Wu}, 1987$ ). For crack length $c<c_{m}$, if

(a)

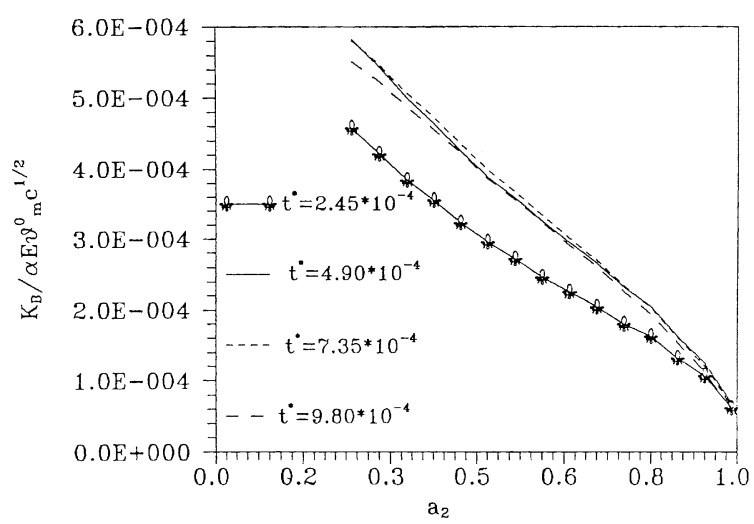

(b)

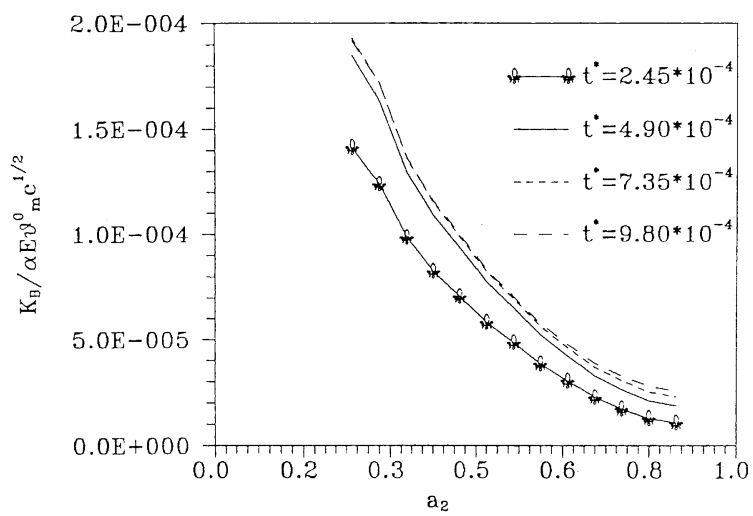

(c)

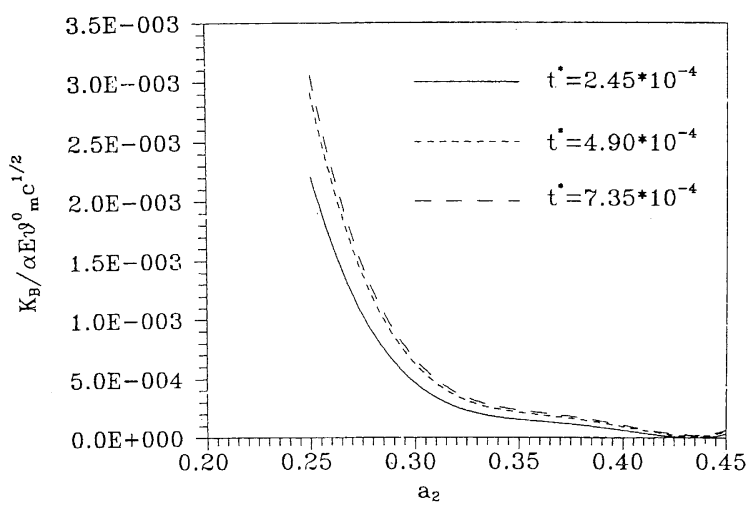

Fig. 7. The normalized transient TSIFs versus $a_{2}$ : (a) shape 1 ; (b) shape 2 ; (c) shape 3 , where $a_{1}=50$ and $r_{0}^{*}=0.3$. 
(a)

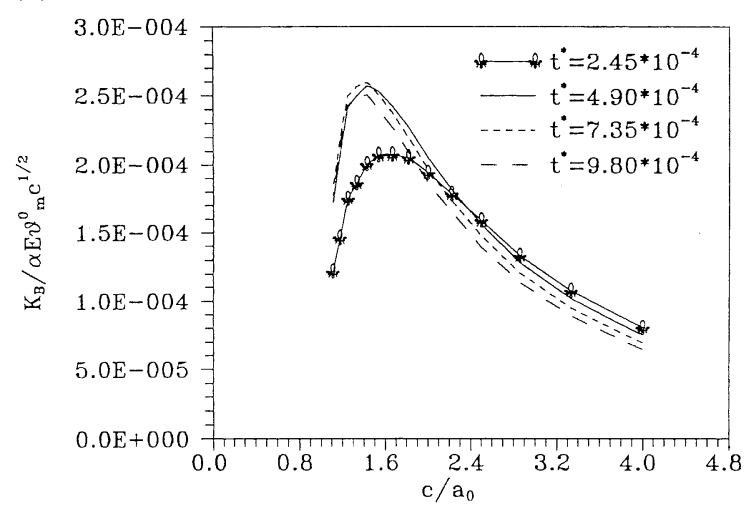

(b)

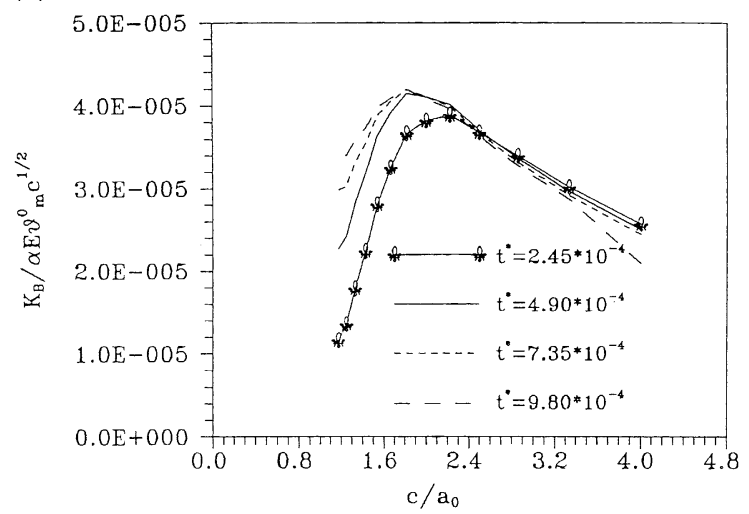

(c)

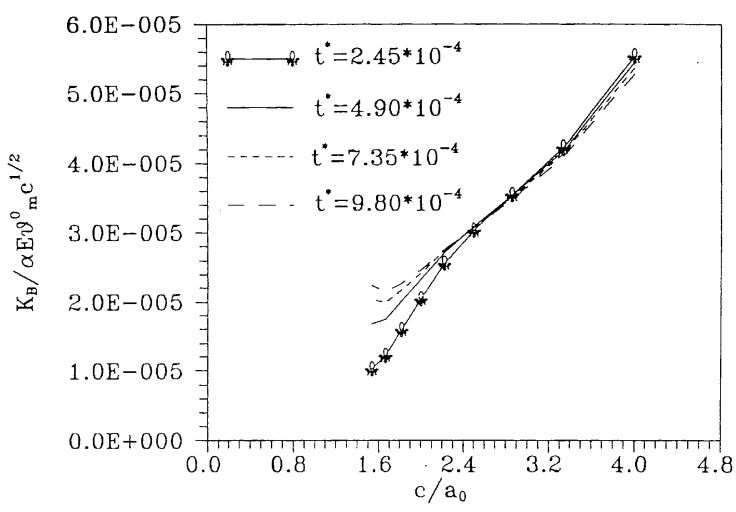

Fig. 8. The normalized transient TSIFs versus the crack length: (a) shape 1; (b) shape 2; (c) shape 3, where $a_{1}=30, a_{0}=0.25$ and $r_{0}^{*}=0.3$.

the critical TSIF is exceeded, the cracks will propagate in an unstable manner, because the TSIF increases until $c=c_{m}$. However, in Fig. 8(c), the cracks always propagate in an unstable manner. The phenomenon of unstable crack-growth was very pronounced for initial notch lengths. This demonstrates that such cracks continued to grow in the region where the static $K(c)$ was below $K_{\mathrm{c}}$. Hence the static crack arrest criterion $K(c) \leqslant K_{\mathrm{c}}$ does not hold in this case, and the crack arrest should be discussed by the dynamic theory of elasticity.

\subsection{The crack surface contact problem}

In Fig. 9, the ratio of the crack surface contact length $2 b$ to the overall crack length $2 c$ is plotted as a function of $t^{*}$. From Fig. 9, one can see that for the shape 1 laser beam, the crack surface contact length will decrease with the increase of time $t^{*}$. However, for the shape 2 laser beam, the crack surface contact length will increase with the increase of time $t^{*}$.

Fig. 10 shows the curves of the crack surface contact length $b / c$ versus normalized time $t^{*}$ for different $a_{2}$. The increase of $a_{2}$ will result in the increase of the crack surface contact length. This corresponds to the decrease of TSIFs with the increase of $a_{2}$ as shown in Fig. 6. It can also be seen that the crack surface 


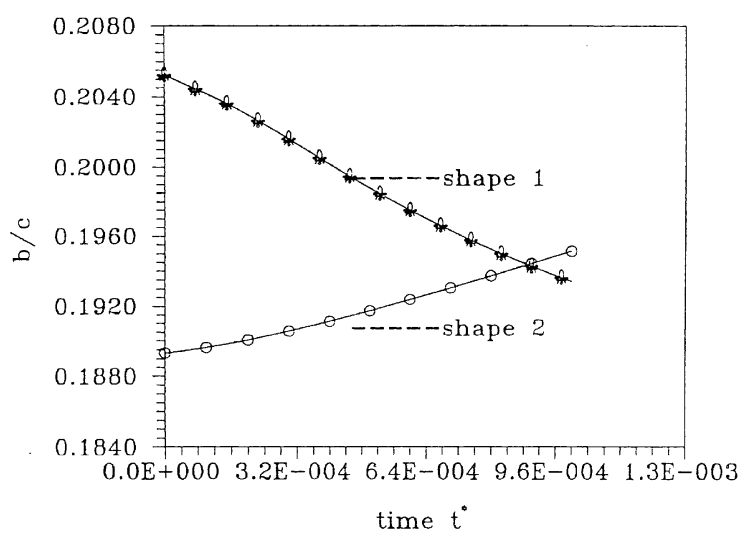

Fig. 9. Crack surface contact length $b / c$ for shapes 1 and 2 laser beam with $a_{2}=0.25$.

(a)

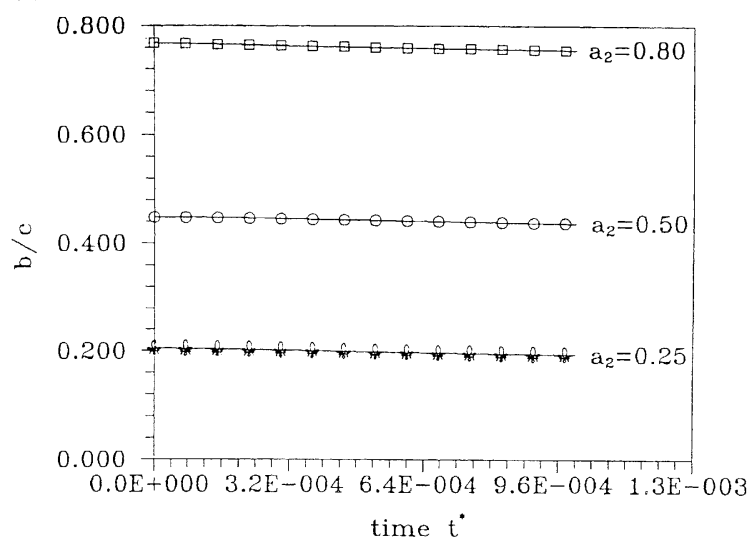

(b)

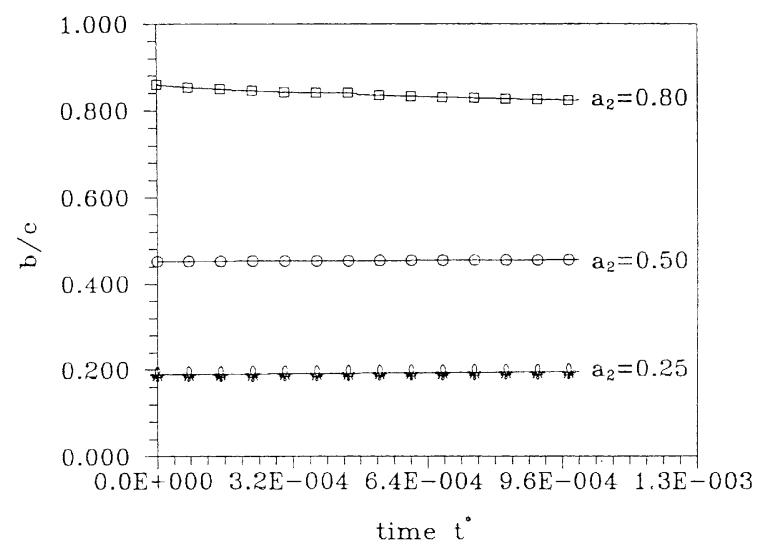

(c)

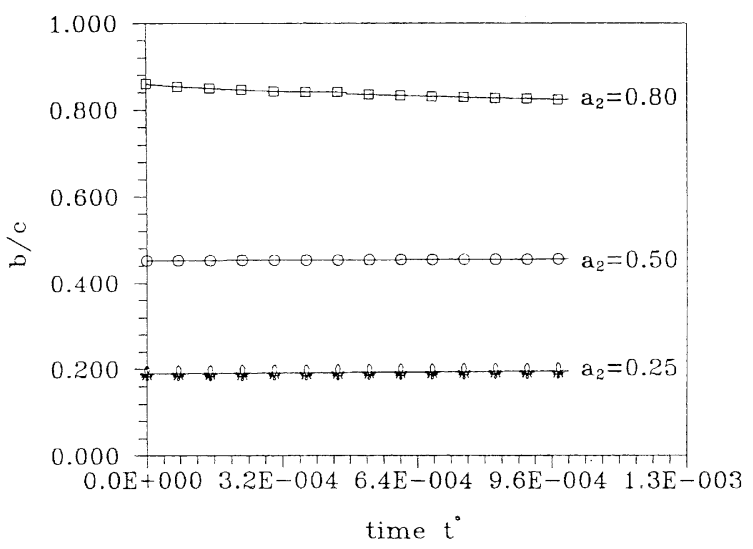

Fig. 10. The curves of crack surface contact length $b / c$ versus normalized time $t^{*}$ for different $a_{2}$ : (a) shape 1 ; (b) shape 2 ; (c) shape 3 , where $r_{0}^{*}=0.3$. 

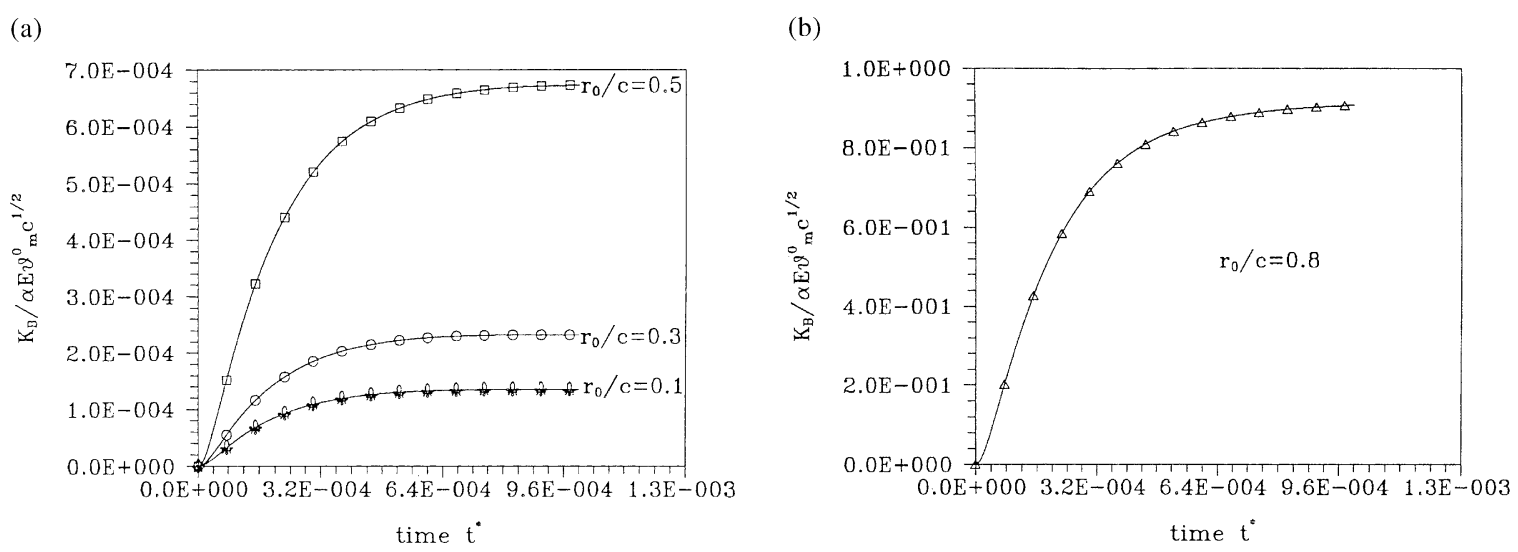

Fig. 11. The dependence of TSIFs on $r_{0} / c$ with $a_{1}=30$ for shape 3 laser beam: (a) $r_{0} / c$ lower than a critical value 0.7 , (b) $r_{0} / c$ larger than a critical value 0.7 .

contact length $b / c$ does not change any more during the laser irradiation. Fig. 11 shows the dependence of parameter $r_{0} / c$ on the transient TSIFs. As $r_{0} / c$ increases the TSIFs increase. If $r_{0} / c$ reaches a critical value 0.7, the TSIFs increase sharply as shown in Fig. 11(b).

\section{Conclusions}

In this paper the characteristics of thermal fracture induced by laser beam thermal shock are investigated. The problem of analyzing this particular transient thermal stress was complicated due to the fact that the crack surfaces will come into contact during thermal shock. The fracture characteristics induced by laser beam thermal shock are in marked different from those induced by mechanical loading. For thermal shock load, the plane of TSIF with crack-length is separated to two regions: stable crack-growth region and unstable crack-growth region. But for general mechanical load, the SIF increases with the square root of the crack length. The dependence of TSIFs on the spatial shape of laser beam is also investigated. One can alter the failure mode in laser-induced material failure by changing the spatial shape of laser beam when its average parameters such as wavelength, intensity, duration and spot size remained unchanged.

\section{Acknowledgements}

The research is supported by NNSF of China (19772043). The collaborative research grant for foreign researchers in Japan was provided to first author Y. C. Z. by JSPS (Japan Society for the Promotion of Science). Their support is gratefully acknowledged.

\section{Appendix A}

In the appendix, some functions are given as,

$$
\begin{aligned}
& F(a)=\frac{K_{\mathrm{r}}}{\sigma_{0} \sqrt{\pi a}} \\
& G(a)=\left[I_{1}(a)-4 F(a) \sqrt{a} I_{2}(a)\right] \frac{\sqrt{a}}{I_{3}(a)}
\end{aligned}
$$




$$
\begin{aligned}
& I_{1}(a)=\pi \sqrt{2} a \sigma_{0} \int_{0}^{a}[F(\xi)]^{2} \xi \mathrm{d} \xi, \quad I_{2}(a)=\int_{0}^{a} \sigma_{\mathrm{r}}(x)(a-x)^{1 / 2} \mathrm{~d} x, \\
& I_{3}(a)=\int_{0}^{a} \sigma_{\mathrm{r}}(x)(a-x)^{3 / 2} \mathrm{~d} x \\
& k_{0}=\sqrt{1-\frac{b^{2}}{c^{2}}}, \quad E\left(k_{0}\right)=\int_{0}^{\pi / 2} \frac{\mathrm{d} \varphi}{\sqrt{1-k_{0}^{2} \sin ^{2} \varphi}}, \quad K\left(k_{0}\right)=\int_{0}^{\pi / 2} \sqrt{1-k_{0}^{2} \sin ^{2} \varphi \mathrm{d} \varphi} \\
& F_{\mathrm{IAr}}(a)=\frac{1}{\sqrt{a b}} \frac{c^{2} \frac{E\left(k_{0}\right)}{K\left(k_{0}\right)}-b^{2}}{\sqrt{c^{2}-b^{2}}} \quad F_{\mathrm{IBr}}(a)=\sqrt{1+\frac{b}{a}} \frac{1}{k_{0}}\left[1-\frac{E\left(k_{0}\right)}{K\left(k_{0}\right)}\right] \\
& u_{\mathrm{rA}}=\frac{\sigma_{0}}{\sqrt{2} E}\left[4 F_{\mathrm{IAr}}(a) \sqrt{a(a-x)}+\frac{G_{\mathrm{IAr}}(a)(a-x)^{3 / 2}}{\sqrt{a}}\right], \\
& u_{\mathrm{rA}}=\frac{\sigma_{0}}{\sqrt{2} E}\left[4 F_{\mathrm{IBr}}(a) \sqrt{a(a-x)}+\frac{G_{\mathrm{IBr}}(a)(a-x)^{3 / 2}}{\sqrt{a}}\right] \\
& G_{\mathrm{IAr}}(a)=\frac{5}{2} a^{-3 / 2} \pi \sqrt{2} \int_{0}^{a} F_{\mathrm{IAr}}^{2}(\xi) \xi \mathrm{d} \xi-\frac{20}{3} \sqrt{a} F_{\mathrm{IAr}}(a), \\
& G_{\mathrm{IBr}}(a)=\frac{5}{2} a^{-3 / 2} \pi \sqrt{2} \int_{0}^{a} F_{\mathrm{IBr}}^{2}(\xi) \xi \mathrm{d} \xi-\frac{20}{3} \sqrt{a} F_{\mathrm{IBr}}(a)
\end{aligned}
$$

In expression (A.6), $x$ is the coordinate with the origin at the B and A for the crack opening displacement $u_{\mathrm{rA}}$ (Fig. 1(e)) and $u_{\mathrm{rB}}$ (Fig. 1(d)), respectively.

\section{References}

Bakioglu, M., Erdogan, F., Hasselman, D.P.H., 1976. Fracture analysis of self-fatigue in surface compression strength glass plates. J. Mater. Sci. 11 (10), 1826-1840.

Berlin, P., Dickman, O., Larsson, F., 1992. Effects of heat radiation on carbon/PEEK, carbon/epoxy and glass/epoxy composites. Composites 23 (4), 235-243.

Blisset, M.J., Smith, P.A., Yeomans, J.A., 1997. Thermal shock behavior of unidirectional silicon cabbide fiber reinforced calcium aluminosilicate. J. Mater. Sci. 32 (2), 317-325.

Boley, B.A., Weiner, J.H., 1985. Theory of Thermal Stresses, Wiley, New York.

Bueckner, H.F., 1970. A novel principle for the computation of stress intensity factors. ZAMM 50, 529-546.

Duan, Z.P., Zhou Y.C., et al., 1994. On laser induced reverse plugging effect. In: Zheng, Z.M., et al., (Eds.), Proceeding of IUTAM Symp. On Impact Dyn., Peking University Press, Beijing, pp. 176-86.

Gupta, T.K., 1972. Strength degradation and crack propagation in thermally shocked $\mathrm{Al}_{2} \mathrm{O}_{3}$. J. Am. Ceram. Soc. 55 (5), $249-253$.

Kagawa, Y., 1997. Thermal shock damage in a two-dimensional SiC/SiC composite reinforced with woven SiC fibers. Compos. Sci. Tech. 57 (5), 607-611.

Oliveira, R., Wu, X.R., 1987. Stress intensity factors for axial cracks in hollow cylinders subjected to thermal shock. Engng. Fract. Mech. 27 (2), 185-197.

Petroski, H.T., Achenbach, J.D., 1978. Computation of the weight function from a stress intensity factor. Engng. Fract. Mech. 10 (2), 257-266.

Rice, J., 1972. Some remarks on elastic crack-tip stress field. Int. J. Solids Struct. 8 (6), 751-758.

Schneider, G.A., Petzow, G., 1991. Thermal shock testing of ceramics - a new testing method. J. Am. Ceram. Soc. 74 (1), $98-102$.

Tada, H., Paris, P.C., Irwin, G.R., 1973. The Stress Analysis of Cracks Handbook, Del Research Corp., Hellertown, PA.

Zhou, Y.C., Duan, Z.P., 1996. Circular brass foil failed by plugging opposite to direction of incident laser beam. Theoretic. Appl. Fract. Mech. 24 (2), 135-138. 
Zhou, Y.C., Duan, Z.P., Yang, Q.B., 1998. Failure of SiC particulate reinforced metal matrix composites induced by laser thermal shock. Metall. Mater. Trans. A 29 (2), 685-692.

Zhou, Y.C., Duan, Z.P., Yang, Q.B., 1999. A thermal elastic analysis on laser-induced reverse bulging and plugging in circular brass foil. Int. J. Solids Struct. 36 (2), 363-389. 